TRANS · núm. I7 2013

NOTA $\cdot 187-193$
Este trabajo quiere presentar una faceta muy poco conocida del intelectual italiano Pier Paolo Pasolini (1922-1975), es decir, la de traductor. En la primera etapa de su vida, transcurrida en Friuli, Pasolini se lanzó a la poesía usando la lengua friulana, con Poesie a Casarsa (1942). Además se dedicó activamente a la afirmación de esta lengua como lengua literaria. En este afán por el reconocimiento y la promoción del friulano, Pasolini otorgaba a la traducción un papel trascendental. Según él, la operación traductora sería la prueba ulterior del estatus del friulano, condición indispensable para darle dignidad de lengua. El trabajo analiza el contexto en que Pasolini realizó las traducciones, y sus reflexiones en torno a esta operación necesaria.

PaLABRAS ClaVE: Pier Paolo Pasolini, lengua friulana, lenguas minoritarias, activismo en traducción.

\title{
Pier Paolo Pasolini y el imperioso afán por traducir
}

Caterina Briguglia

Universitat Autònoma de Barcelona

\section{Pier Paolo Pasolini and the overriding urge to translate}

This study seeks to present a very little known side of the Italian intellectual Pier Paolo Pasolini (1922-1975), that is to say, that of translator. In the first phase of his life, in Friul, Pasolini undertook poetry using Friulan language, with Poesie a Casarsa (1942). Also he actively devoted to the affirmation of this language as a literary language. In promoting and strengthening Friulan language and identity, he gave translation a very important role. According to him, the act of translation was further proof of the status of Friulan and a necessary task he had to carry out to enrich Friulan culture and language. The study analyses the context in which Pasolini carried out these translations, and his thoughts about translation.

KEY WORDS: Pier Paolo Pasolini, Friulan language, minority languages, activism in translation. 
Sin duda es arduo encontrar a un intelectual más prolífico y polifacético que Pier Paolo Pasolini. Sus facetas más conocidas y más estudiadas son las de novelista, poeta, director de cine, y escritor de ensayos sobre el arte, la literatura, la lingüística y la política. Sin embargo, una exploración de su biografía y de la bibliografía recogida alrededor de su nombre nos ofrece la grata sorpresa de que Pasolini fue también dramaturgo, pintor y hasta compositor de canciones. Y es que un estudio de su vida y de su obra nos obliga a adoptar la misma versatilidad, para establecer sugestivas conexiones entre las diferentes formas artísticas, y verlas como partes de un todo coherente, como lenguajes y códigos diferentes que respondían a la misma necesidad de expresarse y la misma lúcida visión del mundo. La actitud apasionada caracteriza a nuestro intelectual en todas y cada una de las actividades que llevó a cabo en su intensísima vida, y ejerció como un imán para todos los amigos que en algún momento lo acompañaron en su camino. Y su legado está, aún hoy en día, treinta y siete años después de su muerte, más vivo que nunca.

Aquí emprendemos la tarea de indagar en otra pasión de nuestro escritor, menos explorada pero no por ello menos significativa: se trata del Pasolini traductor, en la primera fase de su vida, la fase friulana (1942-1950). Cabe advertir que el objetivo de este trabajo no es el estudio descriptivo de las traducciones realizadas por Pasolini, sino la presentación del contexto y de la finalidad por la cual fueron concebidas $y$ publicadas.

\section{PASIÓN Y COMPROMISO CON LA LENGUA FRIULANA}

Pasolini nació en Bolonia en 1922. Sin embargo, pasó toda su juventud en Friul - una región del noreste de Italia- en Casarsa, pue- blo de origen de la madre. Allí entró en contacto con el mundo campesino y experimentó un estilo de vida que se basaba en la contemplación de la naturaleza y el goce provocado por la humilde belleza de las pequeñas cosas. Fue entonces cuando se enamoró de la lengua friulana, ${ }^{\mathrm{I}}$ que para él constituía una forma de expresarse incontaminada, que lo llevaba al mundo arcaico de los campesinos. Sólo por medio de esta lengua era posible expresar los momentos más sentimentales y apasionados de la existencia. El friulano adquiere así para él una dimensión simbólica, casi, como solía afirmar, a la manera de los simbolistas, que empleaban la palabra para alcanzar y expresar una experiencia absoluta.

El friulano no era sólo la lengua de la tierra virgen, sino también la lengua de su madre, y es conocido el vínculo visceral que unía a madre e hijo. De hecho, en casa de Pasolini se hablaba en italiano, y sólo su madre hablaba en dialecto. Es por esta razón que muchos críticos vinculan la elección de adoptar el dialecto como lengua literaria también a la problemática relación entre el autor y su padre, por un lado, y el amor incondicional hacia su madre, por otro. ${ }^{2}$

I El friulano es considerado una lengua por los filólogos, sobre todo gracias a los estudios de uno de los más importantes lingüistas italianos, Graziadio Isaia Ascoli. Sin embargo, Pasolini se refería al friulano utilizando el término «dialecto», para distinguirlo del italiano. Por la misma razón, aquí también adoptamos el término «dialecto», entendido como sistema lingüístico normalmente utilizado en un espacio geográfico limitado y con una reducida producción literaria o escrita. Véase Ascoli (1975) y el ensayo de Lamuela (I987), sobre la historia y el estatus social del friulano, en un estudio en el que esta variedad está asimilada al catalán y al occitano, en cuanto lenguas minoritarias del dominio lingüístico románico que viven dentro de otras comunidades establecidas.

${ }_{2}$ El vínculo entre madre e hijo era tan fuerte que, en su autobiografía en verso, Pasolini afirmó: «La cosa più importante della mia vita è stata mia madre», en Pasolini, «Poeta delle ceneri» [en línea]. 
Finalmente, hay que destacar una tercera y significativa razón que llevó al escritor a usar y a defender el friulano. La contienda lenguadialecto encarnaba también otra oposición fundamental en la biografía del escritor, que era el resultado de un cambio que se había realizado gradualmente y que, después, caracterizó toda su trayectoria vital. Defender el dialecto significaba para él defender un mundo precapitalista, todavía no contaminado por el interés del capital. Por otro lado, el italiano era la lengua de la burguesía, es decir, la clase social que el escritor más odiaba. Y según él era una lengua artificial, que nadie hablaba realmente, una lengua impuesta por el afán de unidad lingüística.

De esta manera, el friulano se convierte en símbolo e instrumento de oposición a la ideología fascista de unidad nacional, entendida también como unidad lingüística, con desprecio de los dialectos. El friulano es la lengua minoritaria que se opone a la homologación cultural impuesta por la dictadura de Mussolini. Desde este punto de vista, la colección Poesie a Casarsa, publicada en I942, es decir, todavía durante el fascismo, representativa de esta etapa friulana del autor, constituyó un verdadero escándalo. Todos los poemas están escritos en friulano, esa lengua que, como todos los dialectos de la península italiana, sufría por parte del régimen un deliberado ostracismo.

Además de su propia producción literaria, Pasolini se dedicaba activamente a la difusión del friulano como lengua literaria. Y por ello fundó, en 1945, junto con otros amigos, la Academiuta di lenga furlana, que miraba a la promoción del dialecto no solamente como lengua oral, sino también como lengua de producción escrita. La ideología que fundamentaba la academia era: «friulanità assoluta, tradizione romanza, influenza delle letterature contemporanee, libertà, fantasia»
(Pasolini, I994: 54), divulgada a través de una revista, Il Stroligut (pequeño almanaque), donde se publicaban composiciones poéticas en friulano, y ensayos de literatura friulana y de lingüística. La Academiuta y su revista nacieron como paladinas no solamente de la lengua friulana, sino más en general, de todas las lenguas minoritarias del área románica. De hecho, en el primer número de Il Stroligut, Pasolini deja claro su interés por considerar el friulano como miembro de la gran familia de las lenguas románicas minoritarias, al declarar: «Il Friuli si unisce, con la sua sterile storia, e il suo innocente, trepido desiderio di poesia, alla Provenza, alla Catalogna, ai Grigioni, alla Rumenia, e a tutte le altre Piccole Patrie di lingua romanza» (I994: 53).

\section{LA TRADUCCIÓN NECESARIA}

Según Pasolini, otro factor clave para la consolidación del friulano era la traducción. En efecto, afirma que «il friulano ha bisogno di traduzioni essendo questo il passo più probatorio di una sua promozione a lingua» (r999a: 282). La operación traductora se convierte en piedra de toque del estado de lengua del friulano, la prueba tangible de su dignidad y riqueza. Por esta razón, invitaba a traducir al friulano, tanto desde las lenguas extranjeras como desde el italiano. Una consideración importante es que, para Pasolini, «non si tratterebbe di ridurre, ma di tradurre; cioè non si tratterebbe di trasferire la materia da un piano superiore (la lingua) a un piano inferiore (il friulano), ma di trasporla da un piano all'altro a parità di livello» (I999a: 282-283). Se confirma, de esta manera, la convicción en la dignidad lingüística del friulano.

La mayoría de las traducciones fueron publicadas en los últimos tres números de la revista

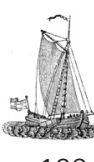

189 
de la Academiuta, entre I945 y I947, y en revistas friulanas de la época. ${ }^{3}$ Se trata en su totalidad de poemas, todos traducidos por el mismo Pasolini, y también por otros miembros de la Academiuta, básicamente por Nico Naldini y Cesare Bortotto. La lista de los principales poetas traducidos es la siguiente: Théodore Aubanel, Juan Ramón Jimenez, Jorge Guillén, Federico García Lorca, Antonio Machado, Thomas Stearns Eliot, William Wordsworth, Friedrich Hölderlin, Stefan George, Georg Trakl, Arthur Rimbaud, Valery Larbaud, Paul Verlaine, Niccolò Tommaseo, Giovanni Pascoli, Giuseppe Ungaretti, Salvatore Quasimodo, y Safo.

Como se puede notar, Pasolini se lanzó a la aventura traductora abarcando desde el principio a un ingente número de poetas y de lenguas de partida (del provenzal, francés, español, inglés, alemán e italiano). Se trata de poetas modernos y la única incursión en el mundo clásico la representa Safo. Cabe decir que, como demuestra Federico Condello (2007), Pasolini tradujo los cuatro fragmentos de Safo usando «con ogni evidenza» una versión italiana moderna traducida por el poeta Quasimodo. Por lo tanto, se trata de un caso de traducción indirecta.

En su artículo sobre el uso de la traducción en la creación de las identidades nacionales, Venuti (2005) afirma que es importante estudiar tanto el desarrollo de las estrategias de traducción adoptadas para traducir un texto como las razones que subyacen a la selección de un autor o de un texto. Como hemos anunciado, aquí no nos detendremos en el análisis de las estrategias de traducción, pero sí que nos interesa saber por qué Pasolini eligió a estos poetas. Efectivamente, la elección no es casual.

3 Hoy las encontramos todas reunidas en la obra magna Tutte le poesie, de Pier Paolo Pasolini (2003).
Podemos identificar tres diferentes razones que pueden haber influido en la elección de los poetas.

I. La primera razón deriva de una afinidad o hermandad poética y estilística con los autores escogidos. La afinidad con el poeta italiano Pascoli es conocida (y de hecho este autor fue el objeto de estudio de la tesis de fin de carrera de Pasolini). Con él compartía, entre otras cosas, la misma actitud de goce ante la belleza de las pequeñas cosas de la vida cotidiana, la añoranza por la ingenuidad de la niñez, y la exaltación de la palabra en su sentido más puro y absoluto. En el caso de los poetas simbolistas franceses y también de los españoles, traductor y traducidos comparten la misma afición por los símbolos, por lo absoluto, una pasión por la naturaleza viva, por lo popular, por una poesía cargada de sensibilidad musical. El mismo Pasolini en más de una ocasión declaró su filiación con los poetas simbolistas, como en la carta a su amigo Franco de Gironcoli, del 3 de noviembre de I945, en la que afirma: «il friulano è il mezzo che ho trovato per fissare ciò che i simbolisti ed i musicisti dell'8oo hanno tanto cercato (...) cioè una 'melodia infinita' o il momento poetico in cui si sente l'infinito nel soggetto». (Pasolini, I986: 210). Y también demostró en varias obras su atracción por España, su literatura y, en particular, su deuda con los grandes poetas españoles. ${ }^{4}$

2. La segunda razón es de carácter estilístico o, mejor dicho, de sofisticación estilística. Si pensamos en Rimbaud, Trakl, George o Ungaretti, salta inmediatamente a la vista la

4 En este sentido, son muy interesantes dos estudios sobre el influjo de la poesía española en la obra pasoliniana: Díaz Pérez (I993) y el más reciente Falchi (20II). Y también el artículo de Mininni (20II), que estudia las traducciones de Juan Ramon Jiménez llevadas a cabo por Pasolini y Naldini, y la relación y coincidencias entre el poeta español y el italiano. 
coincidencia en la profunda sofisticación del texto de partida. Pasolini dio prioridad a autores modernos de singular complejidad, como si quisiera retar las capacidades del friulano. A este propósito declaró: «noi abbiamo tentato traduzioni soprattutto dai moderni, in quanto presentano difficoltà il cui superamento sarebbe stato più probatorio per il friulano, mettendolo a confronto con una esasperata coscienza linguistica» (1999a: 283). En otras palabras, presenciamos «una dimostrazione di virtuosismo perfino provocatoria, una acrobazia nella resa con uno strumento ancora acerbo» (Pellegrini, 2008: 15I), es decir, el friulano.

3. Finalmente, la tercera razón es ideológica y política. Efectivamente, Théodore Aubanel fue un poeta provenzal, activista defensor de la lengua y la literatura provenzales y fundador del grupo literario felibrista; Niccolò Tommaseo fue un dialectólogo italiano de Dalmacia, un poeta y, sobre todo, un patriota, durante los movimientos revolucionarios del siglo XIX contra la monarquía austriaca; finalmente, respecto a García Lorca, además de las afinidades estilísticas, es fácil identificar entre traductor y traducido un común compromiso civil y la misma aversión por la dictadura y por la injusticia, que en el caso del poeta español resultó en su trágica muerte. ${ }^{5}$ Por lo tanto, la elección de los poetas de este tercer grupo ejemplifica la afirmación de Venuti, cuando considera que una de las razones que pueden llevar a la decisión de traducir un texto puede ser «because the social situation in which it was produced is seen as analogous to that of the translating culture and thus as

5 Podemos destacar más razones que pueden haber motivado a Pasolini en la traducción de textos de Lorca. Efectivamente, las afinidades entre los dos poetas son muchas y, en ciertos puntos, trágicas. Véase, a este propósito, el breve ensayo de Jordi Corominas (2009). illuminating of the problems that a nation must confront in its emergence» (Venuti, 2005: 180). Para Pasolini, todosestos poetas encarnan la lucha contra un sistema opresor, ya sea cultural o político.

Como hemos dicho, el acto de traducir era una prueba ulterior del estatus del friulano. La realización de tal operación en sí misma era tan importante que su resultado estético pasaba a un segundo plano. Efectivamente, Pasolini afirma que «la dignità del friulano sarebbe egualmente dimostrata anche da una mediocre riuscita di una simile operazione» (I999a: 283). Con lo cual, lo importante no es el cómo sino el puro hecho de llevarlo a cabo. Esta afirmación confirma el pensamiento de Maria Tymoczko, en su estudio sobre el activismo en traducción, según el cual «nor are activist translations effective primarily because of their form, style or textual strategies» (Tymoczko, 2010: 252). Según la definición de Tymoczko (ib.: I2-I5), se puede hablar de «activismo» en traducción cuando ésta se lleva a cabo como un compromiso social y con objetivos que conllevan una voluntad de acción en la sociedad. Esta acción puede aspirar a un cambio cultural, a la protección y consolidación de una lengua, o también a la construcción o defensa de una identidad. Así las cosas, el uso de la traducción por parte de Pasolini es un ejemplo de activismo en traducción. Efectivamente, él propuso la independencia política y cultural a partir de la lengua, a través de la creación poética y de la traducción.

Por la misma razón llamó su atención el caso de la lengua catalana, víctima como el friulano de la opresión fascista. En I946 conoció a Carles Cardó, poeta catalán exiliado en Friburgo. Pasolini pidió al escritor catalán que le preparase una selección de los mejores poetas catalanes de todos los tiempos. Así, en 1947,

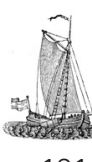

191 
publicó Fiore di poeti catalani en el tercer número de la revista Il Stroligut, que para la ocasión cambió de título y se llamó Quaderno romanzo. Se trata de una selección de nueve poemas (de Joan Rois de Corella, Jacint Verdaguer, Miquel Costa i Llobera, Joan Alcover, Joan Maragall, Josep Carner, Carles Riba, Manuel Bertran i Oriola, y Carles Cardò), traducidos al italiano por Carles Cardò, pero revisados por Pasolini. Definitivamente, representa un ejemplo de traducción activista. Aún más si consideramos que, a mediados del siglo xx, la lengua y la literatura catalanas estaban prohibidas por el régimen franquista. En la nota a la antología, Pasolini destaca la larga y fecunda tradición literaria del catalán, a partir del siglo XIII. Además, alaba la fuerza del pueblo catalán que, a pesar de las adversas vicisitudes históricas, había conseguido preservar su cultura y su lengua, confiriéndole la dignidad de una gran lengua literaria. La lucha por el renacimiento y la independencia de la lengua catalana se encuentra en plena consonancia espiritual con la situación del friulano. El catalán, por lo tanto, constituía un modelo de lengua que los friulanos deberían haber seguido. En definitiva, la traducción de estos poemas en la revista responde al proyecto de importar un modelo de lengua minoritaria que había sabido consolidarse, «una especie de canon o modelo cultural y lingüístico que demostrara la viabilidad de las propuestas lingüísticas y estilísticas que en esos años él mismo [Pasolini] defiende a contracorriente» (Núñez García, 2006: 324). $\mathrm{El}$ autor italiano concluye su nota denunciando la opresión de la dictadura, pero ansiando una situación de esperanza a favor de la autonomía lingüística de Cataluña, puesto que «gli scrittori catalani seguitano a lavorare nelle catacombe in attesa del giorno, forse non lontano, in cui il sole della libertà splenderà di nuovo su questa lingua» (Pasolini, I994: 32).

\section{CONCLUSIÓN}

La fase friulana de Pasolini concluyó en I950, cuando el autor se mudó a Roma. Allí empezó su etapa más prolífica y famosa, es decir, la de novelista y cineasta. El dialecto romano de los suburbios de la capital, código expresivo de la clase social más pobre y olvidada, sustituye al friulano y así una nueva variante lingüística minoritaria se convierte en protagonista de su universo artístico.

En conclusión, en esa etapa la traducción fue un acto necesario para la consolidación de la lengua y la cultura friulanas. La necesidad era de triple índole y el planteamiento, como siempre en el caso de Pasolini, transparente y lúcidamente expuesto: por un lado, la traducción respondió a la necesidad de sentir una comunión estética con poetas de más allá de las fronteras friulanas, y de incorporar y dar a conocer en friulano a autores con la misma sensibilidad poética; por otro, desempeñó el papel de importar un modelo de lengua poética y obligar el friulano, todavía «verde» y con una tradición literaria débil, a poner a prueba sus propias capacidades y superar sus límites lingüísticos; finalmente, como en el caso de la antología catalana, los poemas traducidos ofrecieron una ocasión de protesta política y un modelo de lucha contra la opresión cultural impuesta por las dictaduras. La crítica contra los regímenes dictatoriales y contra la homologación cultural fueron constantes a lo largo de toda la trayectoria vital de Pasolini. Su muerte, y la violencia que la causó, produjo mucha consternación en el mundo intelectual italiano, y la conciencia de haber perdido a un hombre con un extraordinario compromiso con la realidad que lo rodeaba, «no sols un compromís civil, polític [...], sinó més aviat humà, absolut, de donació i de redempció» (García-Medall, en 
Pasolini, 199r: 7). Pero aún duele más pensar que la crueldad humana apagó la voz de este extraordinario intelectual, incansable luchador por la causa de la libertad, en la noche del 2 de noviembre de 1975, justo 23 días antes de la muerte de Franco y del fin de la dictadura en España.

RECIBIDO EN FEBRERO DE 2012 ACEPTADO EN JUNIO DE 2012 VERSIÓN FINAL DE JUNIO DE 2012

\section{REFERENCIAS BIBLIOGRÁFICAS}

Ascoli, G. I. (1975): Scritti sulla questione della lingua, Turín: Einaudi.

Condello, F. (2007): «Pasolini traduttore di Saffo: note di lettura». Testo a fronte, 37, pp. 23-40.

Corominas i Julián, J. (2009): "Pasolini y Lorca, ¿vidas paralelas?» [en línea], Pagine corsare, http://www.pasolini.net/saggistica_PasoliniLorca_Corominas.htm. [Consulta: I4 de febrero de 20I2].

Díaz Pérez, J. C (1993): «Presencia de la cultura española en la obra de Pier Paolo Pasolini». Revista de Filología Románica, ro, pp. 65-84.

Falchi, F. (20II): Pasolini y la cultura española, Barcelona: Editorial Alrevés.

Lamuela, X. (1987): Català, occità, friülà: llengües subordinades $i$ planificació lingüistica, Barcelona: Quaderns Crema.

Mininni, Maria Isabella (20II): «Il giovane Pasolini traduttore di Juan Ramón Jiménez», Hermeneus, I3, pp. I03-I26.
Nuñez García, L. (2006): «Fiore di poeti catalani: Pier Paolo Pasolini y Cataluña». En Assumpta Camps y Lew Zybatow (eds.): La traducción literaria en la época contemporánea: actas de la Conferencia Internacional "Traducción e Intercambio Cultural en la

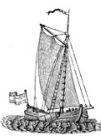
Época de la Globalización», pp. 317-325.

Pasolini, P. P. (I986): Lettere (1940-I954). Nico Naldini (ed.). Turín: Einaudi.

Pasolini, P. P. (I99I): Cartes als amics. Articles de joventut (I94I-I945). Joaquim García-Medall (ed.). Valencia: Universitat de València/ Col·legi universitari de Castellò.

Pasolini, P. P. (I994): L'Academiuta friulana e le sue riviste. Nico Naldini (ed.). Vicenza: Neri Pozza.

Pasolini, P. P. (1999a): Saggi sulla letteratura e sull'arte. Walter Siti y Silvia De Laude (eds.). Con un ensayo de Cesare Segre. Milán: Mondadori.

Pasolini, P. P. (1999b): Saggi sulla politica e sulla società. Walter Siti y Silvia De Laude (eds.). Con un ensayo de Piergiorgio Bellocchio. Milán: Mondadori.

Pasolini, P. P. (2003): Tutte le poesie. Walter Siti y Silvia De Laude (eds.). Milán: Mondadori.

Pasolini, P. P «Poeta delle ceneri» [en linea]. Pagine corsare, http://www.pasolini.net/poesia_ppp_poetaceneri.htm [Consulta: I4 de febrero de 20I2].

Pellegrini, R. (2008): «Classici latini e greci in redazione friulana. Esempi e sondaggi», Incontri triestini di filologia classica 6. Trieste: EUT, pp. I4I-I54.

Tymoczko, M. (ed.) (2010): Translation, Resistance, Activism, Amherst: University of Massachusetts Press.

Venuti, L. (2005): «Local Contingencies: Translation and National Identities». En S. Bermann y M. Wood (eds): Nation, Language, and the Ethics of Translation. Princeton/Oxford: Princeton University Press, pp. $\mathrm{I}_{7} 7^{-202}$. 